\title{
Evaluating the capability of the Sentinel 2 data for soil organic carbon prediction in croplands
}

Fabio Castaldi (1), Andreas Hueni (2), Sabine Chabrillat (3), Kathrin Ward (3), Gabriele Buttafuoco (4), Bart Bomans (5), Kristin Vreys (5), Maximilian Brell (3), and Bas van Wesemael (1)

(1) Earth and LIfe Institute, Université Catholique de Louvain, Louvain la neuve, Belgium (fabio.castaldi@ uclouvain.be), (2) Department of Geography, Remote Sensing Laboratories, University of Zurich, Zurich, Switzerland (ahueni@ geo.uzh.ch), (3) Helmholtz-Zentrum Potsdam, Deutsches GeoForschungsZentrum GFZ, Potsdam, Germany (chabri@ gfz-potsdam.de), (4) Institute for Agricultural and Forest Systems in the Mediterranean, National Research Council of Italy (CNR), Rende (CS), Italy (gabriele.buttafuoco@cnr.it), (5) Flemish Institute for Technological Research - VITO, Mol, Belgium ( bart.bomans@vito.be)

The short revisit time of the Sentinel-2 (S2) constellation entails a large availability of remote sensing data, but S2 data have been rarely used to predict soil organic carbon (SOC) content. Thus, this study aims at comparing the capability of multispectral S2 and airborne hyperspectral remote sensing data for SOC prediction, and at the same time, we investigated the importance of spectral and spatial resolution through the signal-to-noise ratio (SNR), the variable importance in the prediction (VIP) models and the spatial variability of the SOC maps at field and regional scales. We tested the capability of the S2 data to predict SOC in croplands with quite different soil types and parent materials in Germany, Luxembourg and Belgium, using multivariate statistics and local ground calibration with soil samples. We split the calibration dataset into sub-regions according to soil maps and built a multivariate regression model within each sub-region. The prediction accuracy obtained by S2 data is generally slightly lower than that retrieved by airborne hyperspectral data. The ratio of performance to deviation (RPD) is higher than 2 in Luxembourg (2.6) and German (2.2) site, while it is 1.1 in the Belgian area. After the spectral resampling of the airborne data according to S2 band, the prediction accuracy did not change for four out of five of the sub-regions. The variable importance values obtained by S2 data showed the same trend as the airborne VIP values, while the importance of SWIR bands decreased using airborne data resampled according the S2 bands. These differences of VIP values can be explained by the loss of spectral resolution as compared to APEX data and the strong difference in terms of SNR between the SWIR region and other spectral regions. The investigation on the spatial variability of the SOC maps derived by S2 data has shown that the spatial resolution of S2 is adequate to describe SOC variability both within field and at regional scale. 